\title{
microRNA-203 Modulates Wound Healing and Scar Formation via Suppressing Hes1 Expression in Epidermal Stem Cells
}

\author{
Ziheng Zhou ${ }^{\mathrm{a}} \quad$ Bin Shu ${ }^{\mathrm{a}}$ Yingbin $\mathrm{Xu}^{\mathrm{a}}$ Jian Liu ${ }^{\mathrm{a}}$ Peng Wang ${ }^{\mathrm{a}}$ Lei Chen ${ }^{\mathrm{a}}$ \\ Jingling Zhao ${ }^{a}$ Xusheng Liu ${ }^{a}$ Shaohai $\mathrm{Q}^{\mathrm{a}}$ Kun Xiong ${ }^{\mathrm{b}}$ Jun Wu $\mathrm{Wu}^{\mathrm{a}}$ Julin Xie \\ aDepartment of Burn Surgery, First Affiliated Hospital of Sun Yat-Sen University, Guangzhou, \\ bDepartment of Anatomy and Neurobiology, School of Basic Medical Sciences, Central South \\ University, Changsha, China
}

\section{Key Words}

Epidermal stem cells $\cdot \mathrm{miR}-203 \cdot$ Hes $1 \cdot$ Wound healing $\bullet$ Scar

\begin{abstract}
Background/Aims: Little is known how miR-203 is involved in epidermal stem cells (ESCs) differentiation and scar formation. Methods: We first used luciferase assay to determine the interaction of miR-203 with the 3'-UTR in regulation of Hes1 expression. We then used flow cytometry to analyze the effects of miR-203 expression on the differentiation of ESCs to MFB by determination of CK15 ratio and $\alpha$-SMA. To confirm the results of flow cytometry analysis, we used Western blot to examine the expression of $\alpha$-SMA, Collagen I (Col I), and Collagen III (Col III), as well as the expression of Notch1, Jagged1, and Hes1 in ESCs after the treatment of pre-miR-203 or anti-miR-203. Finally, we examined the effects local anti-miR-203 treatment on would closure and scar formation using a mouse skin wound model. Results: Pre-miR-203 treatment increased ESCs differentiation to MFB cells, as indicated by decreased CK15 ratio and increased MFB biomarkers. This phenomenon was reversed by overexpression of Hes1 in ESCs. In addition, skin incision increased expression of miR-203 in wound tissue. Local treatment of anti-miR-203 could accelerate wound closure and reduce scar formation in vivo, which was associated with increased re-epithelialization, skin attachment regeneration, and collagen reassignment. Finally, we confirmed that anti-miR-203 treatment could inhibit ESCs differentiation in vivo via increasing Hesl expression. Conclusion: Taken together, our results suggested that overexpression of miR-203 in ESCs after skin wound may be a critical mechanism underlying the scar formation.
\end{abstract}




\section{Cellular Physiology Cell Physiol Biochem 2018;49:2333-2347

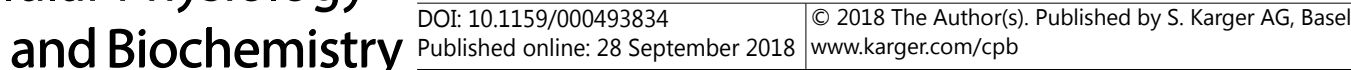 \\ Zhou et al.: MicroRNA-203 and Epidermal Stem Cells}

\section{Introduction}

One of the most common complications during wound healing is the formation of scars. The adverse physiological and psychological effects that are associated with scars formation following the healing of wounds remain a major medical problem for patients $[1,2]$. While various therapies are available for preventing scar formation during skin wound healing, their effects are still unsatisfactory $[3,4]$. Therefore, it is necessary to study the mechanisms of scar formation and develop novel therapies.

Growing evidence has shown that lack of epidermal stem cells (ESCs) and the excessive hyperplasia of myofibroblasts (MFB) are two important factors in scar formation [5-7]. ESCs possess strong proliferation and differentiation potential, and are located in the basal layer of the epidermis and the follicle bugle of hair [8]. Under physiological conditions, ESCs are involved in maintaining the normal structure and function of skin and play a critical role in wound repairing via proliferation, migration, and differentiation [9]. Previous study has shown that scar tissue exhibits lower number of ESCs and higher number of MFB than normal skin [10]. More recently, we demonstrated that this phenomenon is dependent on the activation of Notch/Jagged1 signaling pathway [11, 12]. Activation of the Notch1/ Jagged1 pathway can promote ESC proliferation and inhibit the cells' differentiation to MFB $[13,14]$. Specifically, upon the binding of Jagged1 to Notch1 receptors, the proteolytic cleavage will induce the release of the Notch intracellular domain (NICD), causing it being translocated to the nucleus and resulting in the activation of Hairy/Enhancer of split-1 gene (Hes1). Importantly, it has been shown that Hes1 plays a vital role in promoting proliferation and inhibiting differentiation of ESCs [15].

However, skin wound healing involves multiple cellular signaling. Many studies have identified various miRNAs involved in cell differentiation and skin wound healing $[16,17]$. One of these important miRNAs is miR-203. Previous studies have shown that miR-203 exhibits potent antiproliferative function [18-20] and is involved in regulating the balance between stem cell proliferation and terminal differentiation in skin cells, by targeting p63 when stem cells in the epidermis are proliferating and differentiating into stratified epithelium [21]. miR-203 has the highest level of expression in the skin [22]. It induces cell cycle exit and represses "stemness" in epidermal progenitors, suggesting its involvement in keratinocyte differentiation [21, 23]. Importantly, the expression of miR-203 is drastically enhanced in the wound surrounding tissue [24-27]. Recent studies have shown that miR203 can suppress Hesl expression [28]. Based on these results, we hypothesized that miR-203 may play a critical role in scar formation by suppressing Hes 1 expression in the ESCs. Therefore, we designed the study to determine whether miR-203 could regulate the proliferation and differentiation of ESCs by modulating Hes1 expression and ultimately regulate wound healing and scar formation.

\section{Materials and Methods}

\section{Animals}

Eight-week-old C57BL/6 male and female mice (ratio = 1:1) were purchased from Shanghai Laboratory Animal Center and were housed in the Animal Resource Facility under $12 \mathrm{~h} \mathrm{light/dark} \mathrm{cycle} \mathrm{(lights} \mathrm{on/off} \mathrm{at}$ $7 \mathrm{am} / 7 \mathrm{pm}$ ) and had free access to food and water. All procedures and experiments involving animals in the present study were performed in accordance with the National Institutes of Health Guide for Care and Use of Laboratory Animals (Institute of Laboratory Animal Resources, Commission on Life Sciences 2011). All animal experiments were approved by the Institutional Animal Care and Use Committee of First Affiliated Hospital of Sun Yat-Sen University, China. 


\section{Cellular Physiology Cell Physiol Biochem 2018;49:2333-2347

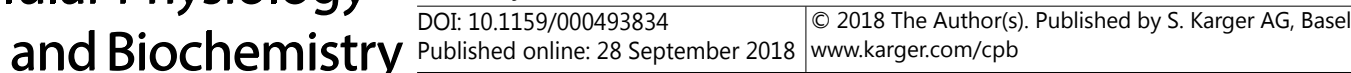 \\ Zhou et al.: MicroRNA-203 and Epidermal Stem Cells}

\section{Isolation of ESCS}

We carefully dissected skin samples from the back of 8-week-old C57BL/6 male and female mice from other tissue. The skin samples were then cut into small pieces (approximately $0.3 \times 0.3 \mathrm{~cm}^{2}$ ). Skin samples were then incubated in PBS containing 0.5\% Dispase II (17105041; Gibco, Shanghai, China) at $4{ }^{\circ} \mathrm{C}$ overnight. We then carefully dissected the epidermal sheets from the dermis and placed the epidermal sheets in $0.25 \%$ trypsin (25200-056; Gibco, Shanghai, China) for digestion at $37{ }^{\circ} \mathrm{C}$ for 20 minutes. Dulbecco's modified Eagle's medium (DMEM, 12100-046; Gibco, Shanghai, China) containing 10\% FBS was then added in to inactivate the trypsin. Samples were then subjected to filtering and centrifuge. Cells were resuspended in keratinocyte serum-free medium (K-SFM, 17005042; Gibco, Shanghai, China) and seeded at a density of $10^{5}$ cells $/ \mathrm{cm}^{2}$ in flasks, which were coated with $100 \mu \mathrm{g} / \mathrm{ml}$ collagen IV (ab6586; Abcam, Shanghai, China). Cells were allowed to adhere to the flasks for 15 minutes at $37^{\circ} \mathrm{C}$. The adhering cells were then collected and cultured in $\mathrm{K}-\mathrm{SFM}$ medium in $5 \% \mathrm{CO}_{2}$ at $37^{\circ} \mathrm{C}$. Upon the culture reached $70-80 \%$ confluence, cells were digested and passaged at a ratio of 1:2 [29]. To characterize the cells, cells were evaluated with markers, including integrin- $\alpha 6^{\text {bri }}$ (3750S; Cell Signaling Technology, Shanghai, China) and CD71 dim (553264; BD biosciences, Shanghai, China) by immunofluorescence staining [30].

\section{Luciferase assay}

The full-length Hes1 $3^{\prime}$-UTR was first cloned downstream of the sequence that encodes luciferase via XbaI site using pGL3-Basic Vector (Takara, Shanghai, China). We then used a site-directed mutagenesis method to introduce the mutation of a miR-203 binding site into the luciferase construct. The used primers were listed in Table 1. We conducted the luciferase assay using HEK293T cells in the 24-well culture plates. HEK293T cells were plated for 24 hours, followed by transfection with pEF-control or pEF-miR-203 expression vector, pGL3-Basic, wild type Hes1 3'-UTR or mutated 3'-UTR vectors. The transfection control was pTk-Renilla. Transfection was performed using Lipofectamine LTX (Invitrogen, Shanghai, China) based on manufacturer's protocol. All experiments were conducted in triplicates, and all the experiments were repeated at least 3 times.

\section{mRNA half-life assay}

In this experiment, we used NIH3T3 cells. Twenty-four hours before the assay, pEF-control or pEFmiR-203 expression vector was used to transfect NIH3T3 cells, followed by Actinomycin-D treatment. Cells were then collected at different time points $(0,20,40,60,90,120,150$ and $180 \mathrm{~min})$. Trizol was used to extract RNA based on manufacturer's protocol (Invitrogen, Shanghai, China). Superscript reverse transcription and quantitative realtime PCR (qRT-PCR) were then carried out to detect endogenous expression levels of Hes1 mRNA. The expression levels of Hes1 mRNA were normalized to internal control, GAPDH. qRT-PCR was performed as described below. Primers are listed in Table 1.

\section{Viral production}

Mouse-specific Hes1 lentiviral construct (LV-Hes1) was purchased from Abm (LV524028; Abmgood, Shanghai, China). Recombinant lentivirus was produced by transient transfection of HEK293T cells according to Tronolab protocols. Briefly, subconfluent HEK293T cells were co-transfected with $20 \mu \mathrm{g}$ of transfer vector, $15 \mu \mathrm{g}$ of packaging plasmid (psPAX2) and $6 \mu \mathrm{g}$ of envelope plasmid (pMD2.G). Two days later, supernatant was

Table 1. List of Primers

\begin{tabular}{|c|c|c|}
\hline Primer name & Sequences & Application \\
\hline \multicolumn{3}{|l|}{ Pri-miR-203 } \\
\hline \multirow[t]{2}{*}{$\mathrm{F}$} & TTATGCCGGCTAGATCCATAGACAGCAACAG & \multirow{3}{*}{ Amplification of Pri-miR-203 } \\
\hline & & \\
\hline $\mathrm{R}$ & GCCGGTTTAAACGTTAGAAAGGGCCCTGGATC & \\
\hline \multicolumn{3}{|c|}{ Hes1 3'UTR mut } \\
\hline $\mathrm{F}$ & ATGTGATGCGAATGTTTGTTTGAAAATGC & \multirow{3}{*}{ Mutation of Hes1 3'UTR } \\
\hline & & \\
\hline $\mathrm{R}$ & GCATTTTCAAACAAACATTCGCATCACAT & \\
\hline \multicolumn{3}{|l|}{ Hes1 } \\
\hline $\mathrm{F}$ & TGAAGGATTCCAAAAATAAAATTCTCTGGG & \multirow{3}{*}{ RT-PCR } \\
\hline & & \\
\hline $\mathrm{R}$ & CGCCTCTTCTCCATGATAGGCTTTGATGAC & \\
\hline \multicolumn{3}{|l|}{ Gapdh } \\
\hline $\mathrm{F}$ & ATCTTCTTGTGCAGTGCCAGCCTCGTCCCG & \multirow{3}{*}{ RT-PCR } \\
\hline & & \\
\hline $\mathrm{R}$ & AGTTGAGGTCAATGAAGGGGTCGTTGATGG & \\
\hline \multicolumn{3}{|l|}{ GFP } \\
\hline $\mathrm{F}$ & ACGTAAACGGCCACAAGTTC & \multirow{3}{*}{ RT-PCR } \\
\hline & & \\
\hline $\mathrm{R}$ & AAGTCGTGCTGCTTCATGTG & \\
\hline
\end{tabular}




\section{Cellular Physiology Cell Physiol Biochem 2018;49:2333-2347 \begin{tabular}{ll|l} 
and Biochemistry & DOI: 10.1159/000493834 & $\begin{array}{l}\text { O } 2018 \text { The Author(s). Published by S. Karger AG, Basel } \\
\text { www.karger.com/cpb }\end{array}$ \\
\cline { 2 - 3 }
\end{tabular} \\ Zhou et al.: MicroRNA-203 and Epidermal Stem Cells}

ultra-centrifuged in Beckman L-70 at $26,000 \mathrm{rpm}$ at $4{ }^{\circ} \mathrm{C}$ for $2 \mathrm{~h}$. Viral pellet was then resuspended in 100 $\mu \mathrm{l}$ PBS. Fresh viral suspension $(20 \mu \mathrm{l})$ was used for each infection.

\section{Establishment of ESCs with stable expression of Hes1}

ESCs from C57/BL6 mice were transduced with Hes1 lentiviral constructs as previously described [31]. ESCs were infected with lentiviral vector overexpressing Hes1 with GFP signals (over $80 \%$ of transfected cells) were selected for further experiments.

miR-203 overexpression and knockdown

We ordered synthetic pre-miR-203, anti-miR-203 and scrambled negative control RNAs from Shanghai GenePharma Co., Ltd., (Shanghai, China). The cells were seeded in 6-well plates or 60-mm dishes. Upon the cells reached approximately $70 \%$ confluency, the cells were transfected with Lipofectamine 2000 (Invitrogen, Shanghai, China). In each well, equal amounts $(20 \mathrm{ng} / \mathrm{mL})$ of pre-miR-203, anti-miR-203 or scrambled negative control RNA were used. Twenty-four hours after transfection, we harvested the cells for subsequent quantitative RT-PCR and Western blotting experiments. The transfection success rate was about $82 \%$.

\section{Flow cytometry analysis}

ESCs $\left(1 \times 10^{6} / \mathrm{ml}\right)$ were trypsinized and suspended in $2 \%$ BSA/PBS (16000-044; Gibco, Shanghai, China) after 10 days of culture. Following centrifuge and resuspension, we incubated the cells with the following primary antibodies: anti-CK10 (1:100, ab9026; Abcam, Shanghai, China), anti-CK15 (1:100, ab52816; Abcam, Shanghai, China), and anti- $\alpha$-SMA (1:20, ab32575; Abcam, Shanghai, China) for 2 hours at room temperature. Cells were then subjected to centrifuge and washing, and FITC-labeled secondary antibody IgG (1:500, ab6785; Abcam, Shanghai, China) was then added in the resuspended cells incubated for 30 minutes. BD Accuri C6 (BD Biosciences, Shanghai, China) was then used to detect the expression of CK15, CK10, and $\alpha$-SMA. All experiments were performed in triplicate.

\section{Protein extraction and western blot analysis}

We extracted total proteins of ESCs using the ProteoPrep® Total Protein Extraction Kit (PROTTOT$1 \mathrm{KT}$; Sigma-Aldrich, Shanghai, China), and determined the protein concentration using the BCA Assay Kit. Protein samples were boiled for 10 minutes, protein extract $(50 \mu \mathrm{g})$ were then loaded in $10 \%$ SDS-PAGE gels for electrophoresis at $100 \mathrm{~V}$ for 2 hours, followed by transferring to PVDF membranes (Millipore, Shanghai, China) at $100 \mathrm{~V}$ for 90 minutes. The membranes were then blocked with $5 \%$ nonfat milk in TBST $(0.1 \mathrm{M}$, $\mathrm{pH}=7.4 \mathrm{f}$ and incubated at $4{ }^{\circ} \mathrm{C}$ overnight with one of the following primary rabbit anti-mouse antibodies: anti- $\alpha$-SMA (1:1, 000, ab32575; Abcam, Shanghai, China), anti-Collagen I (1:1, 000, ab34170; Abcam, Shanghai, China), anti-Collagen III (1:1, 000, ab7778; Abcam, Shanghai, China), anti-Jagged1 (1:500, ab7771; Abcam, Shanghai, China), anti-Notch1 (1:1, 000, ab52627; Abcam, Shanghai, China), and anti-Hes1 (1:1, 000, ab71559; Abcam, Shanghai, China). The membranes were then washed with with TBS/Tween-20 solution, and incubated with peroxidase-conjugated secondary antibody IgG (1:2, 000, ab6721; Abcam, Shanghai, China). Odyssey infrared imaging system (LI-COR Biosciences, Shanghai, China) was used to detect protein bands and the images were analyzed by Image Pro-Plus 6.0 software (Media Cybernetics). Protein levels were normalized to GAPDH. All experiments were performed in triplicate.

\section{Skin wound model}

We anesthetized mice using $1 \%$ pentobarbital $(30 \mathrm{mg} / \mathrm{kg})$ and shaved the hair on the back. We then made a circular, full-thickness skin excision $(10 \mathrm{~mm}$ in diameter $)$ in the middle of back of the spine. After skin excision, anti-miR-203 (10 $\mu$ g dissolved in $100 \mu \mathrm{L}$ of PBS) was directly injected into the surrounding dermis of the wound at three sites once daily for 14 days. The control wounds received equal amount of scrambled negative control. We recorded wound healing using photographed images. Digital planimetry was used to measure wound area with ImageJ software version 2.1.4.6 (NIH, Bethesda, MD). The wound residual rate and scar index were calculated using the formula as described previously [32]. 


\section{Cellular Physiology Cell Physiol Biochem 2018;49:2333-2347 and Biochemistry Published online: 28 September 2018 le $\begin{aligned} & \text { (c) } 2018 \text { The Author(s). Published by S. Karger AG, Basel } \\ & \text { www.karger.com/cpb }\end{aligned}$ \\ Zhou et al.: MicroRNA-203 and Epidermal Stem Cells}

\section{Quantitative real-time PCR}

Total RNA was extracted from the wound tissue samples using Trizol Reagent (Invitrogen, Shanghai, China), and then transcribed into cDNA using the PrimeScript RT reagent Kit (Takara, Shanghai, China). Quantitative real-time PCR (qRT-PCR) was conducted in the Stratagene Mx3000P real-time PCR system (Agilent Technologies, CA, USA) using SYBR Premix ExTaq (Takara, Shanghai, China) and the primer sequences for miR-203 were shown as follows: forward, 5'-GTGCAGGGTCCGAGGT-3'; reverse, 5'-GCCGCGTGAAATGTTTAGG-3'. GAPDH (ab9485; Abcam, Shanghai, China) was used as internal control. The relative expression ratio of mRNA was calculated using the $2^{-\Delta \Delta C T}$ method. All the experiments were repeated 3 times.

\section{Histological analysis and immunohistochemistry staining}

Skin tissue samples were fixed with formalin, embedded in paraffin, and sectioned at $4 \mu \mathrm{m}$ thickness. The sections were deparaffinized and stained with hematoxylin and eosin (H\&E) and Masson, and were evaluated under standard light microscopy (OLYMPUS, Japan) by experimenter who were blinded to the experiment conditions. To label $\alpha$-SMA, we blocked the sections using 2\% goat serum in PBS for 20 minutes and then incubated the sections with mouse monoclonal anti- $\alpha$-SMA (1:50, ab7817; Abcam, Shanghai, China) at $4{ }^{\circ} \mathrm{C}$ overnight. Sections were then washed in PBS, followed by incubation with an HRP-conjugated secondary antibody (1:2, 000, ab97051; Abcam, Shanghai, China) for 1 hour at room temperature. The sections were further incubated with 2, 4-diaminobenzidine substrate and counterstained with hematoxylin.

\section{Immunofluorescence analysis}

Sections were first washed in PBS, followed by blocking in 10\% goat serum (16210064; Gibco, Shanghai, China) at $37^{\circ} \mathrm{C}$ for 30 minutes. To conduct double labeling, we added two compatible primary mouse anti-rabbit antibodies: anti-BrdU (1:250, ab8152; Abcam, Shanghai, China) and anti- $\alpha$-SMA (1:200, ab7817; Abcam, Shanghai, China), and anti-Hes1 (1:100, ab119776; Abcam, Shanghai, China), and incubated the sections at $4{ }^{\circ} \mathrm{C}$ overnight. On the next day, the sections were washed with $3 \% \mathrm{BSA} / \mathrm{PBS}$, followed by incubation with secondary antibodies for 1 hour: goat anti-mouse IgG labeled with Alex Fluor 488 (1:200, ab150113; Abcam, Shanghai, China) and goat anti-mouse IgG labeled with Alexa Fluor 594 (1:200, ab150116; Abcam, Shanghai, China). Images were captured using a fluorescence microscope (OLYMPUS, Japan).

\section{Statistical analysis}

We analyzed all data using PRISM5.0 software (GraphPad, CA, USA). Values were expressed as the mean \pm standard deviation (SD). Because there was no difference in all data analysis between male and female groups, we combined male and female group data together. Student's $t$ test was used to compare the difference between control and experimental groups. One-way analysis of variance (ANOVA) was used for comparing the differences between multiple groups. A Bonferroni post-hoc test for pairwise comparisons was performed as appropriate. $P<0.05$ indicates that the difference was statistically significant.

\section{Results}

MiR-203 regulated Hes1 expression by interacting with 3'-UTR of Hes1 mRNA

We first determined the role of interaction of miR-203 with the 3'-UTR in regulation of Hes1 expression. We examined the luciferase reporter expression in the presence or absence of a miR-203 binding site of the 3'-UTR of Hes1 mRNA (Fig. 1A). We transfected HEK293T cells with control or miR-203 expression vector together with the luciferase reporters. Luciferase reporter containing SV40 late 3'-UTR was used as a control. When there was a wild-type 3'-UTR of Hes1 mRNA, the expression of reporter was down-regulated by miR-203. However, this effect was not seen after the miR-203-binding site in the 3'-UTR of Hes1 mRNA was mutated (Fig. 1B and C). These results suggested that miR-203 negatively regulates the expression of Hes1 via interacting with the 3'-UTR of Hes1 mRNA. 


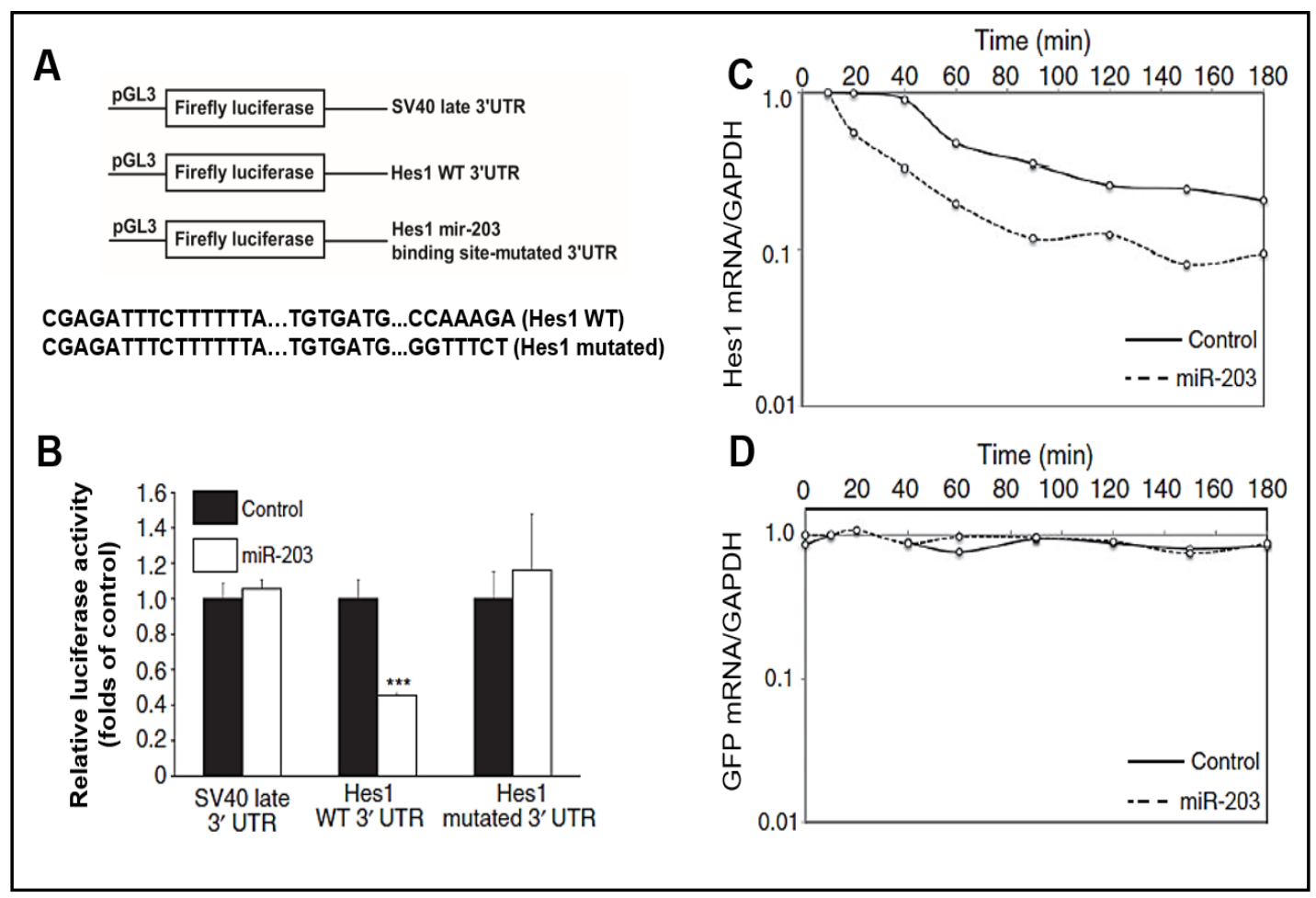

Fig. 1. microRNA-203 targets the 3'-UTR of Hes1 mRNA and regulates Hes1 expression. (A) The schematic structures of luciferase reporters containing SV40 late 3'-UTR, the wild-type (WT) Hes1 3'-UTR or the Hes13'UTR whose miR-203-binding site was mutated. (B) Luciferase assay with the control or miR-203 expression vector in 293T cells. MiR-203 repressed the expression of the reporter containing the WT Hes1 3'-UTR but not mutated Hes1 3'-UTR or SV40 late 3'-UTR. The averages with SD of three independent experiments are shown. ${ }^{* * *} P<0.001, t$-test. (C) Hes1 mRNA stability assay. (D) GFP mRNA expression in GFP vectortransfected NIH3T3 cells in the presence of the control or miR-203 expression vector was quantified by qRTPCR. The value at each time point was the average of three independent experiments. Each was performed in triplicates.

\section{MiR-203 promoted the differentiation of ESCS to MFB in vitro}

ESCs were treated with synthetic pre-miR-203, anti-miR-203 or scrambled negative control RNAs for 10 days. To determine ESC differentiation to MFB, we examined the expression of $\alpha$-SMA, CK10, and CK15 using flow cytometry. The ratio of CK15 and CK10 reflects the purity of ESCs $[33,34]$ and $\alpha$-SMA is a specific marker of MFB $[35,36]$. We found that treatment of pre-miR-203 significantly reduced the ratio of CK15 compared with the control group $(P<0.05$; Fig. $2 \mathrm{~A}$ and $\mathrm{B})$. We confirmed that these manipulations significantly altered the expression levels of miR-203 in ESCs (Fig. 2C). However, when ESCs were pretransduced with LV-Hes1, the effects of pre-miR-203 on the ratio of CK15 were not seen. Similarly, we found that treatment of pre-miR-203 significantly increased the percentage of $\alpha$-SMA positive cells compared with the control group $(P<0.05$; Fig. 3A and B). However, when ESCs were pre-transduced with LV-Hes1, the effects of pre-miR-203 treatment on the percentage of $\alpha$-SMA positive cells were not seen. These results indicated that miR-203 could promote the differentiation of ESC to MFB in vitro.

\section{MiR-203 promoted the formation of MFB in vitro}

To confirm the results of flow cytometry, we also examined the expression of $\alpha$-SMA, Collagen I (Col I), and Collagen III (Col III) using western blot analysis. Col I and Col III are metabolites of MFB, and are also valid markers for MFB [35,36]. We found that the expression of $\alpha$-SMA, Col I, and Col III was significantly lower after anti-miR-203 treatment compared with 


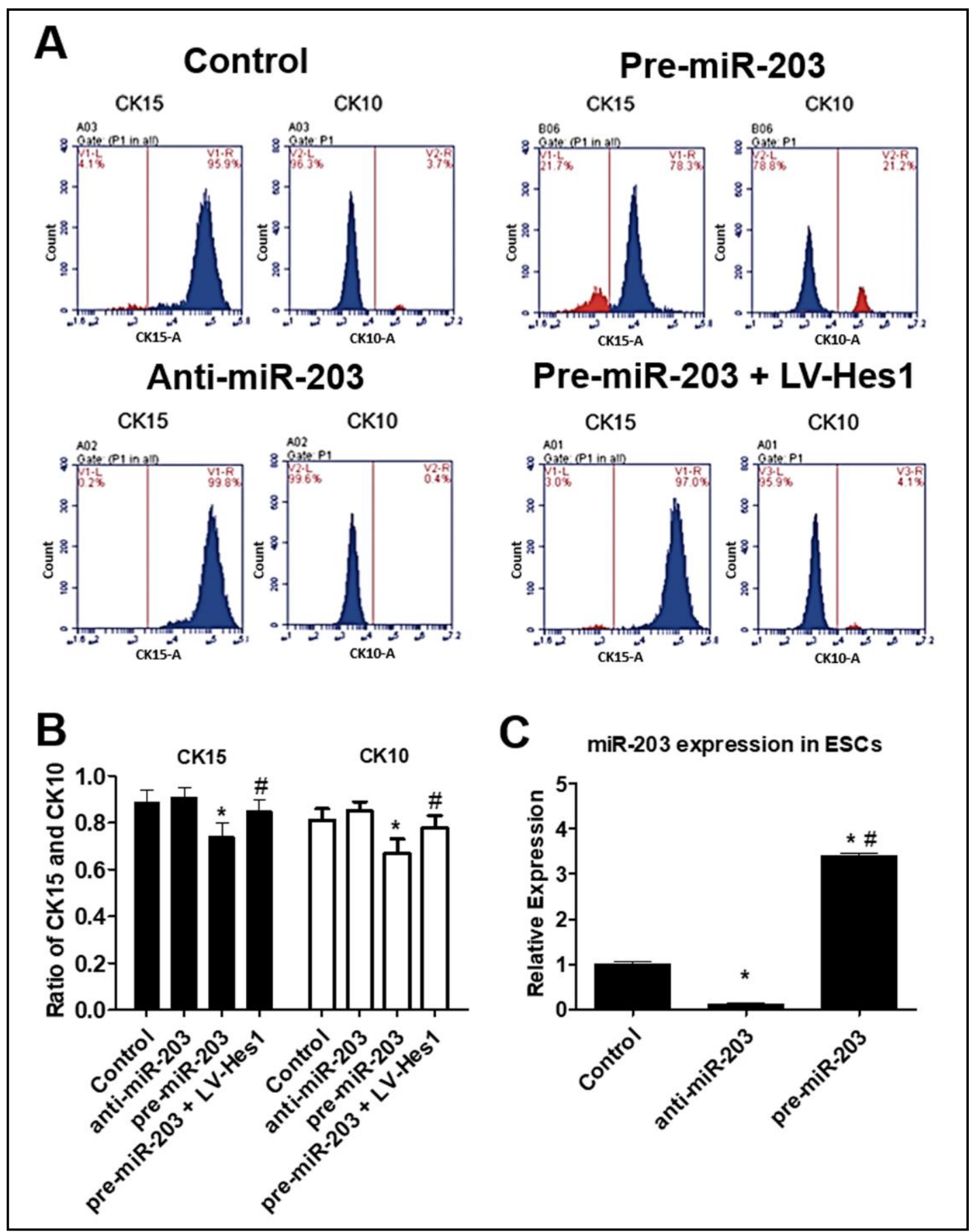

Fig. 2. Flow cytometry analysis of CK10 and CK15 expression in ESCs. (A) Expression of CK10 and CK15 of each treatment group on day 10 detected by flow cytometry analysis. (B) Ratio of CK15 and CK10 of each treatment group on day 10. (C) Relative expression of miR-203 in ESCs. ${ }^{*} P<0.05$ compared with control, \#P<0.05 compared with pre-miR-203. 
control $(P<0.05$; Fig. 4A). Furthermore, pre-miR-203 treatment resulted in higher levels of expression of $\alpha$-SMA, Col I, and Col III in ESCs compared with control $(P<0.05$; Fig. 4A). Additionally, when ESCs were pre-transduced with LV-Hes1, the effects of premiR-203 on the expression of $\alpha$-SMA, Col I, and Col III in ESCs were not seen. To test the effects of miR-203 on Notch1/Jagged1-Hes1 signaling pathway, we also examine the expression of Notch1, Jagged1, and Hes1 in ESCs. We found the expression of Jagged1 or Notch1 was not altered by these treatments. However, we found that anti-miR-203 reduced the expression of Hes1, and pre-miR-203 increased the expression of Hes1 in ESCs $(P<0.05$; Fig. 4B). Additionally, when ESCs were pre-transduced with LV-Hes1, the effects of pre-miR-203 on the expression of Hes1 in ESCs were reversed $(P<0.05$; Fig. 4B). Taken together, our results not only confirmed that Hes1 as a downstream molecule of Notch1/Jagged1 pathway plays a critical role in ESCs differentiation to MFB, but also indicated that miR-203 can interact with Notch1/Jagged1 pathway to modulate the ESCs differentiation to MFB.

Ant $i-m i R-203$ treatment accelerated wound closure and reduced scar formation in vivo

To investigate the role of miR-203 in wound healing and scar formation in vivo, we conducted our experiments using a mouse skin wound model. Mice were treated with either anti-miR-203 or scramble negative control RNA. During the period of wound healing, wound
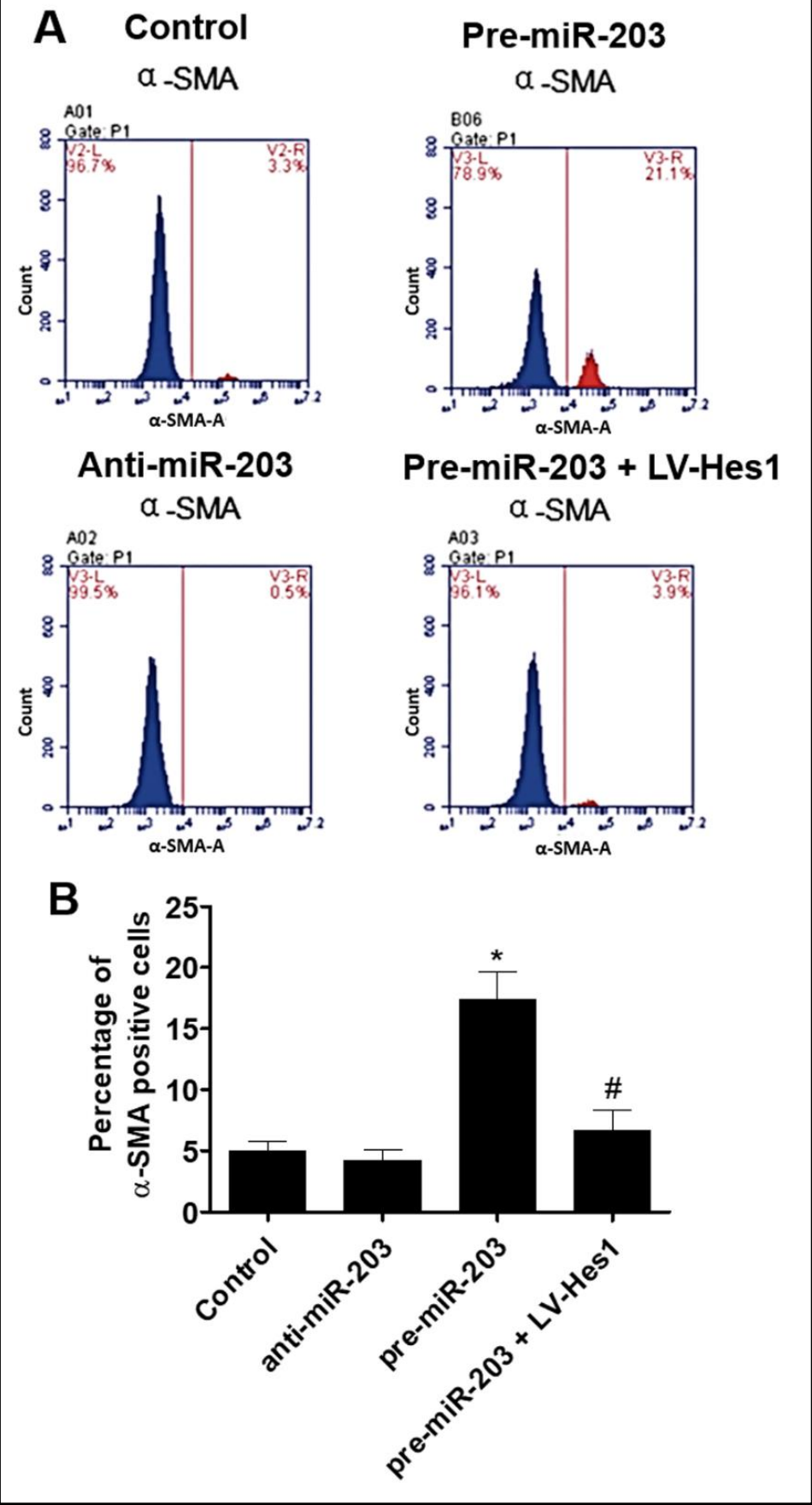

Fig. 3. Flow cytometry analysis of $\alpha$-SMA expression in ESCs. (A) Expression of $\alpha$-SMA of each treatment group on day 10 detected by flow cytometry analysis. (B) Percentage of $\alpha$-SMA positive cells of each treatment group on day $10 .{ }^{*} P<0.05$ compared with control, $\# P<0.05$ compared with pre-miR-203. 


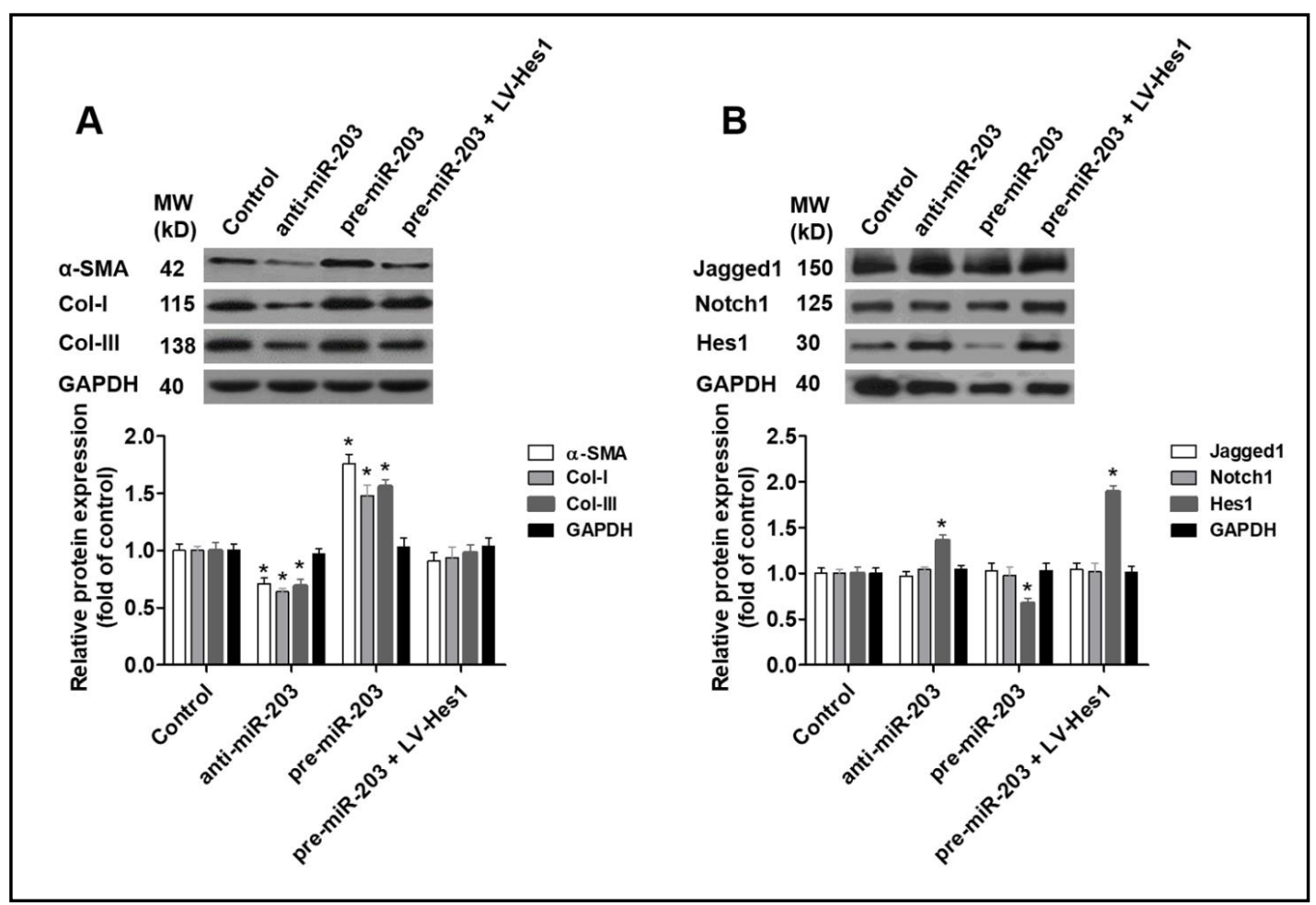

Fig. 4. miR-203 promoted ESCs differentiation to MFB by enhancing Hes1 expression in vitro. (A) Representative immunoblot results showing relative protein levels of $\alpha$-SMA, Col I, and Col III for each treatment group on day 10. (B) Representative immunoblot results showing relative protein levels of Jagged1, Notch1, and Hes1 for each group on day 10. ${ }^{*} P<0.05$ compared with control.

healing time and the images of the wound for each animal was recorded. Wound areas and the thickness of scar tissues were also measure. We calculated the residual wound area rate and scar index. We found that skin incision increased the expression of miR-203 in wound tissues across time as measured by qRTPCR $(P<0.05$; Fig. 5). Furthermore, antimiR-203 treatment reduced the healing time (data not shown), and decreased skin thickness $(P<0.05$; Fig. 6A) scar index $(P<0.05$; Fig. 6B), and the residual wound area $(P<0.05$; Fig. 6C; data not shown), compared with control. These results suggested that miR-203 overexpression after skin wound could delay wound healing and promote scar formation.

\section{Anti-miR-203 treatment promoted re-epithelialization, skin attachment regeneration, and collagen reassignment}

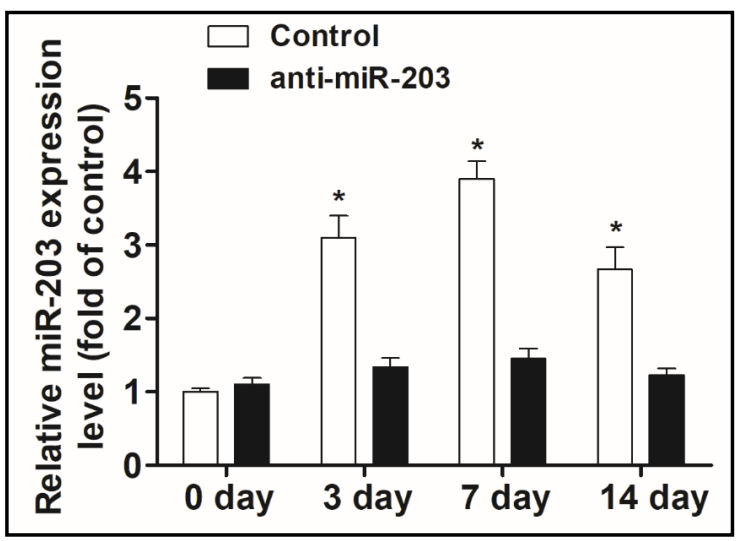

Fig. 5. Effects of skin incision on miR-203 expression in wound surrounding tissue. Skin incision induces miR203 expression in wound surrounding tissue. AntimiR-203 local treatment suppressed the expression of miR-203 over 14 days ${ }^{*} P<0.05$ compared with day 0 $(n=10)$.

We further evaluated the wound healing quality and assessed the scar formation. We 
collected the scar tissue at the end of experiments (day 14). We evaluated re-epithelialization, skin appendage regeneration, and collagen reassignment using $\mathrm{H} \& \mathrm{E}$ and Masson staining. We found that the quality of wound healing after antimiR-203 treatment was increased compared with control group. Specifically, anti-miR-203 treatment resulted in increased cell layers, epidermal ridges, formation of primitive hair follicle, sweat gland structures, and collagen arrangement (Fig. 6D). These results suggested that miR-203 plays a critical role in regulating re-epithelialization, skin appendage regeneration, and collagen reassignment.

Anti-miR-203 treatment inhibited differentiation of ESCs to MFB in vivo

We labeled ESCs with BrdU and detected the relative expression levels of Hes1 and $\alpha$-SMA at the same time by double-immunofluorescence staining. We found that anti-miR-203 treatment significantly increased the percentage of Hes1/ BrdU double-positive cells $(P<0.05$; Fig. 7A and B) compared with control. On the contrary, anti-miR-203 treatment significantly decreased the percentage of $\alpha$-SMA/BrdU double-positive cells $(P<0.05$; Fig. 7C and D) compared with control. These results suggested that miR-203 could promote ESC differentiation to MFB by enhancing Hes1 expression.
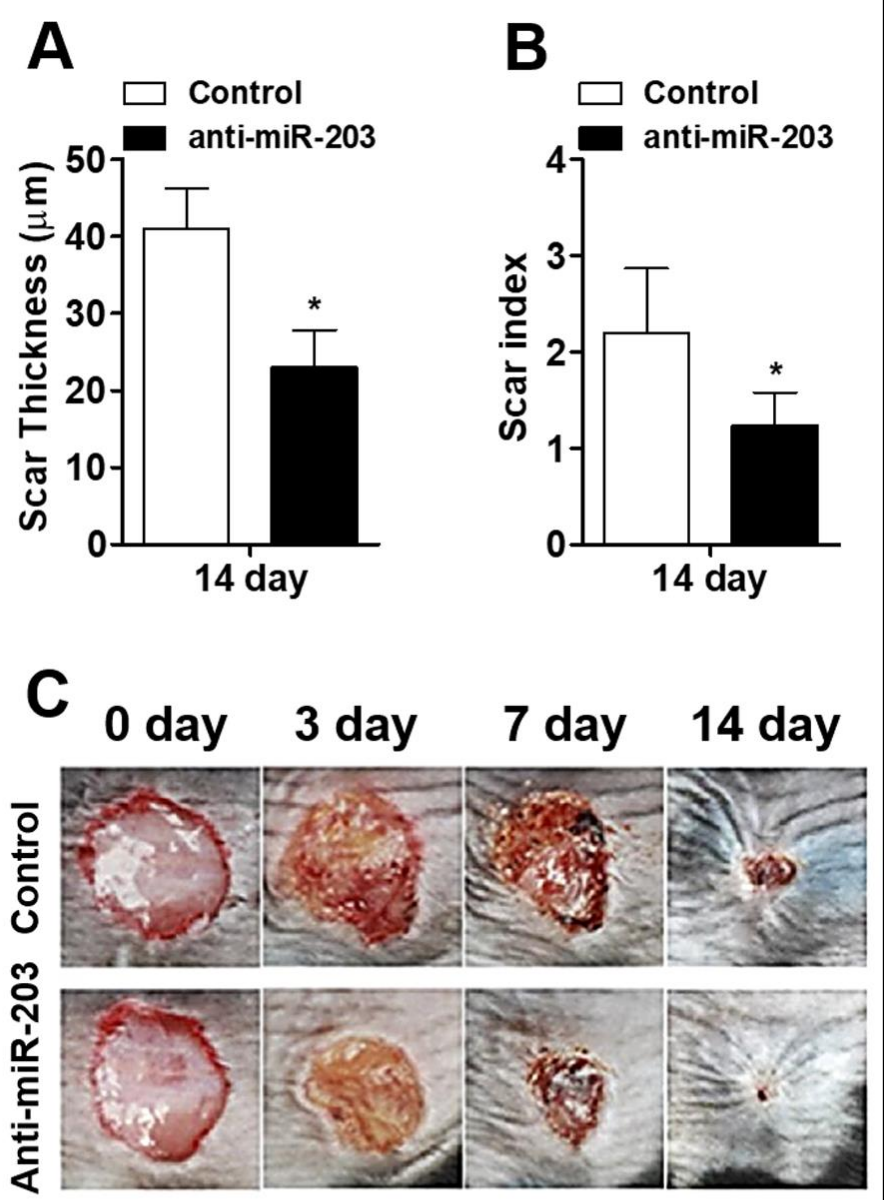

D

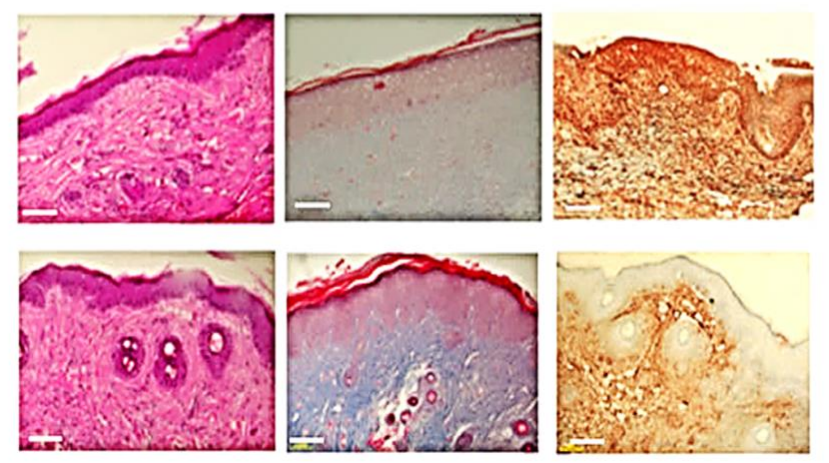

Fig. 6. Anti-miR-203 treatment accelerated wound closure and reduced scar formation in vivo. (A) Scar thickness at day $14 .{ }^{*} P<0.05$ compared with control $(n=10)$. (B) Scar indexes for each group at day 14. ${ }^{*} P<0.05$ compared with control $(\mathrm{n}=10)$. (C) Representative skin wound images from each treatment group taken on post-injury days $0,3,7$, and 14. (D) Skin tissue sections stained with H\&E and Masson and immunohistochemical staining for $\alpha$-SMA. 


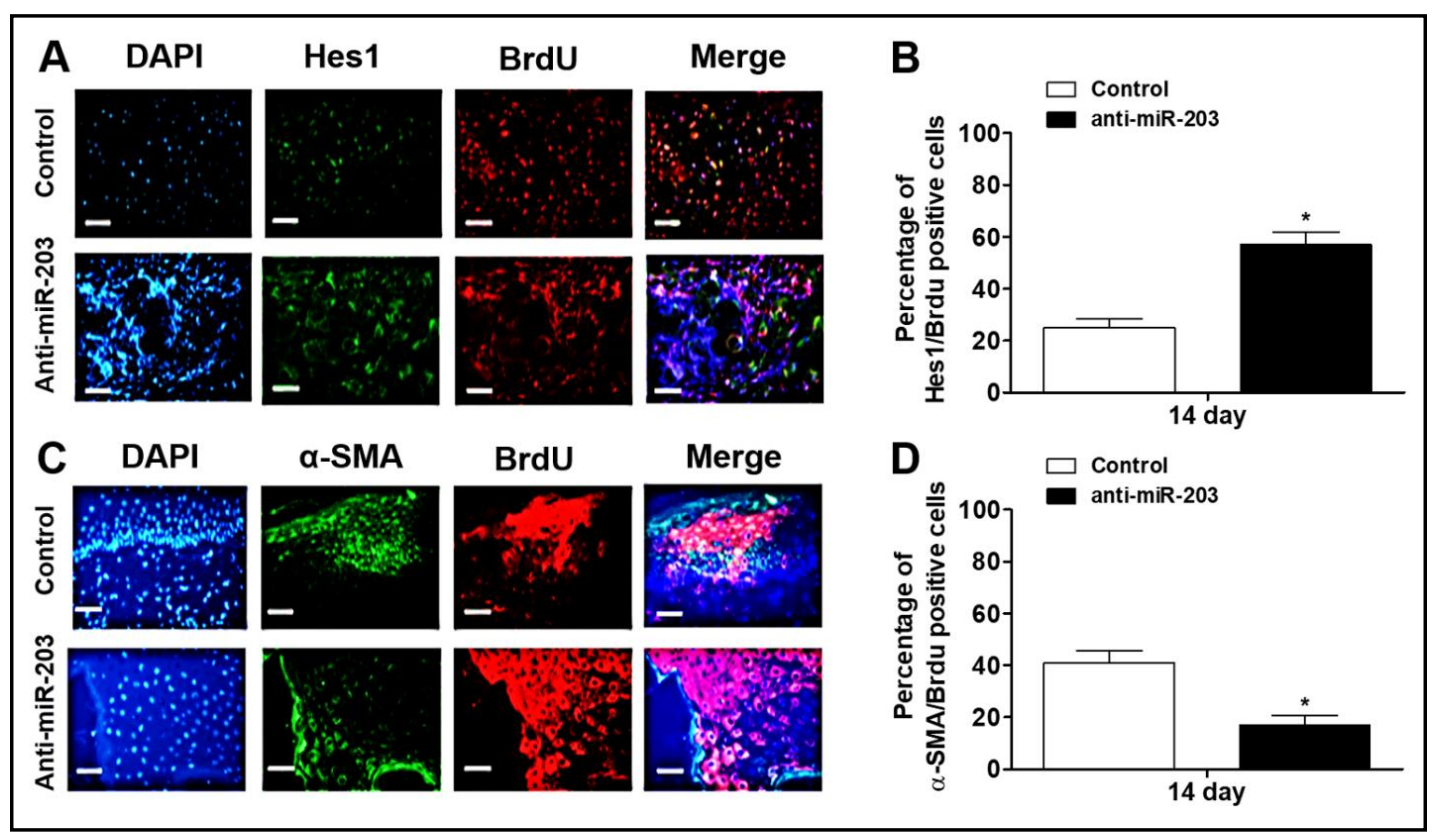

Fig. 7. Effects of anti-miR-203 treatment on Hes1 expression and differentiation of ESCs to MFB in healed skin. (A) Representative Brdu/Hes1 double-positive cells in healed skin and (B) the percentage of the positive cells to total cells in healed skin of each group on post-injury day 14. (C) Representative Brdu/ $\alpha$ SMA double-positive cells in healed skin and (D) the percentage of the positive cells to total cells in healed skin of each group on post-injury day 14 . Student's $t$ test, ${ }^{*} P<0.05$ compared with control value $(\mathrm{n}=10)$.

\section{Discussion}

The present study was designed to examine the role of miR-203 in ESCs differentiation to MFB and skin wound healing and scar formation. Consistent with previous study, we found that Hes1 expression is negatively regulated by miR-203, which interacts with the 3'-UTR of Hes1 mRNA. Furthermore, using flow cytometry analysis, we showed that premiR-203 treatment increased the percentage of MFB cells, as indicated by MFB biomarkers, including $\alpha$-SMA and CK15. This phenomenon was reversed in ESCs that has overexpression of Hes1 via lentiviral transduction (LV-Hes1). We further confirmed these results by using Western blot analysis, and demonstrated additional biomarkers of MFB, including Col-1 and Col-III, were also increased by pretreatment of pre-miR-203. Similarly, this effect was reversed in LV-Hes1 transduced ESCs. These results indicated that miR-203 could promote the differentiation of ESC to MFB in vitro via suppressing Hes1 expression. To examine the role of miR-203 in skin wound healing and scar formation, we first found that skin incision increased expression of miR-203 in wound tissue. We applied anti-miR-203 locally and found that anti-miR-203 treatment after skin incision could accelerate wound closure and reduce scar formation in vivo. This effect was associated with increased re-epithelialization, skin attachment regeneration, and collagen reassignment. Finally, we confirmed that antimiR-203 treatment could inhibit differentiation of ESCs to MFB in vivo via increasing Hesl expression. Taken together, our results suggested that overexpression of miR-203 in ESCs after skin wound may be a critical mechanism underlying the scar formation.

Our previous study has shown that the Notch1/Jagged1 pathway plays a key role in ESCs differentiation to MFB. Hes1 is an essential downstream signaling molecule of Notch1/ Jagged1 pathway [37]. It has been demonstrated that the activation of Notch1/Jagged1 pathway can result in the activation of its downstream gene Hes1, and can promote stem cell proliferation and inhibit their differentiation [38]. In our previous study, we demonstrated that activation of the Notch1/Jagged1 pathway contributes to promoting ESC proliferation 


\section{Cellular Physiology Cell Physiol Biochem 2018;49:2333-2347

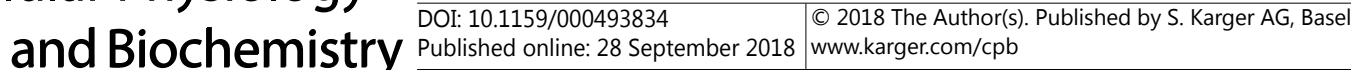 \\ Zhou et al.: MicroRNA-203 and Epidermal Stem Cells}

and inhibiting ESC differentiation [13]. Adding to this literature, the present study first demonstrated that miR-203 may be a paralleling signaling pathway in regulation of ESCs differentiation to MFB. miRNAs are 22-nucleotide-long endogenously expressed noncoding RNAs. Many studies have shown that miRNAs can regulate gene expression by inhibition of translation and/or transcription in animals. miRNAs are critically involved in various physiological and pathological processes, and are implicated in a number of diseases [39]. Growing evidence indicates that dysregulation of specific miRNA is associated with wound healing processes [25, 40,41]. Specifically, studies have demonstrated that some miRNAs play pivotal roles in skin development and the changes of morphology and function $[25,42]$. Previous studies have examined miRNA expressions in mice without Dicer or dgcr8 keratinocytes, and reported that these mice exhibited depletion of ESCs, dysfunction of skin barrier, disorder of hair growth and development, and hyperplasia of basal follicular keratin cells $[43,44]$. It has been shown that miR-203 is the most abundant keratinocyte-specific miRNA in the epidermis and plays a critical role in cell proliferation and differentiation. MiR203 is induced in the skin concomitantly with stratification and differentiation [40]. When miR-203 was overexpressed in the basal layer, the proliferative capacity of cells was reduced earlier, and cell-cycle existence was initiated. In contrast, depletion or knockout of miR-203 can regulate the transition of keratinocytes from the basal layer to the upper layers [45]. Expression of miR-203 is increased in patients with chronic inflammatory skin disease such as psoriasis and atopic eczema, suggesting that miR-203 is involved in inflammatory reaction, cell apoptosis and protease activity [46-48]. Furthermore, miR-203 expression during cells differentiation was described as a 'stemness' repressor and can promote differentiation process $[26,49]$, suggesting that miR-203 can act as a switch between cell proliferation and differentiation. However, studies have also shown that miR-203 was not expressed in proliferating and migrating keratinocytes at the edge of a wound, but was highly expressed in the wound's surrounding [50]. Consistent with these results, our study demonstrated that skin incision induced miR-203 expression in the wound surrounding tissues. Logically, we applied local treatment of anti-miR-203 in the wound surrounding tissue. We reported that anti-miR-203 could accelerate wound closure and reduce scar formation in vivo. This effect was associated with increased re-epithelialization, skin attachment regeneration, and collagen reassignment, likely due to inhibition of differentiation of ESCs to MFB.

In addition to miR-203, several other miRNAs, such as miR-21, miR-34, and miR$146 \mathrm{a} / \mathrm{b}$ are also involved in regulation of cell differentiation and proliferation in skin development [51-54]. Therefore, a reasonable hypothesis is that dysregulation of miRNA expression critically contributes to the wound healing processes and scar formation. While the present study was focused on examining the role of miR-203 in skin wound healing and scar formation, future studies will be necessary to evaluate the role of miRNAs in regulation of wound healing and scar formation comprehensively. This line of research will not only be important for understanding the mechanisms of scar formation, but also be critical for identification of effective therapeutic targets that can promote wound healing process without scar formation.

\section{Acknowledgements}

This paper was supported by the National Natural Science Foundation of China (Grant No. 81671935) and Science and Technology Planning Project of Guang Dong Province, China (Grant No. 2017A040405026) and Guangzhou Science and Technology Project (Grant No. 2018-0402-SF-0686).

\section{Disclosure Statement}

The authors have no conflicts of interest to disclose.

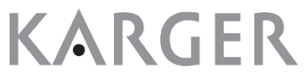




\section{Cellular Physiology Cell Physiol Biochem 2018;49:2333-2347 \begin{tabular}{l|l|l} 
and Biochemistry Published online: 28 September 2018 & $\begin{array}{l}\text { (c) } 2018 \text { The Author(s). Published by S. Karger AG, Basel } \\
\text { www.karger.com/cpb }\end{array}$ \\
\hline
\end{tabular}

\section{References}

1 Martin P: Wound healing--aiming for perfect skin regeneration. Science 1997;276:75-81.

2 Diegelmann RF, Evans MC: Wound healing: an overview of acute, fibrotic and delayed healing. Front Biosci 2004;9:283-289.

3 Berman B, Maderal A, Raphael B: Keloids and hypertrophic scars: pathophysiology, classification, and treatment. Dermatol Surg 2017;43:S3-S18.

4 Berman B, Perez OA, Konda S, Kohut BE, Viera MH, Delgado S, Zell D, Li Q: A review of the biologic effects, clinical efficacy, and safety of silicone elastomer sheeting for hypertrophic and keloid scar treatment and management. Dermatol Surg 2007;33:1291-1303.

5 Zhang G-Y, Li X, Chen X-L, Li Z-J, Yu Q, Jiang L-F, Ding J, Gao W-Y: Contribution of epidermal stem cells to hypertrophic scars pathogenesis. Med Hypotheses 2009;73:332-333.

6 Li-Tsang CW, Feng B, Huang L, Liu X, Shu B, Chan YT, Cheung K-K: A histological study on the effect of pressure therapy on the activities of myofibroblasts and keratinocytes in hypertrophic scar tissues after burn. Burns 2015;41:1008-1016.

-7 Hu MS, Rennert RC, McArdle A, Chung MT, Walmsley GG, Longaker MT, Lorenz HP: The Role of Stem Cells During Scarless Skin Wound Healing. Adv Wound Care (New Rochelle) 2014;3:304-314.

-8 Charruyer A, Ghadially R: What's new in dermatology: epidermal stem cells. Giornale italiano di dermatologia e venereologia: organo ufficiale, Societa italiana di dermatologia e sifilografia 2011;146:5767.

-9 Blanpain C, Fuchs E: Epidermal homeostasis: a balancing act of stem cells in the skin. Nat Rev Mol Cell Bio 2009;10:207-217.

10 Zhou S, Cai J, Niu F, Zong X, Xu J, Du L, Chen G: Comparison of biological characteristics and quantity of epidermal stem cells from hypertrophic scar skin and normal skin of human beings. Zhonghua yi xue za zhi 2014;94:1097-1100.

11 Shu B, Yang R, Shi Y, Xu Y, Liu J: Notch1 Signaling Regulates Wound Healing via Changing the Characteristics of Epidermal Stem Cells. J Stem Cell Res Ther 2016;6:2.

12 Wang P, Shu B, Xu Y, Zhu J, Liu J, Zhou Z, Chen L, Zhao J, Liu X, Qi S: Basic fibroblast growth factor reduces scar by inhibiting the differentiation of epidermal stem cells to myofibroblasts via the Notch1/Jagged1 pathway. Stem Cell Res Ther 2017;8:114.

13 Yang R-H, Qi S-H, Shu B, Ruan S-B, Lin Z-P, Lin Y, Shen R, Zhang F-G, Chen X-D, Xie J-L: Epidermal stem cells (ESCs) accelerate diabetic wound healing via the Notch signalling pathway. Bioscience Rep 2016;36:e00364.

14 Liu J, Sato C, Cerletti M, Wagers A: Chapter twelve-notch signaling in the regulation of stem cell selfrenewal and differentiation. Curr Top Dev Biol 2010;92:367-409.

15 Yeo S-Y, Chitnis AB: Jagged-mediated Notch signaling maintains proliferating neural progenitors and regulates cell diversity in the ventral spinal cord. P Natl Acad Sci USA 2007;104:5913-5918.

-16 Coskunpinar E, Arkan H, Dedeoglu BG, Aksoz I, Polat E, Araz T, Aydos A, Oztemur Y, Akbas F, Onaran I: Determination of effective miRNAs in wound healing in an experimental Rat Model. Cell Mol Biol (Noisy-legrand) 2015;61:89-96.

17 Wang W, Yang C, Wang XY, Zhou LY, Lao GJ, Liu D, Wang C, Hu MD, Zeng TT, Yan L, Ren M: MicroRNA-129 and -335 Promote Diabetic Wound Healing by Inhibiting Sp1-Mediated MMP-9 Expression. Diabetes 2018;67:1627-1638.

18 Liu T, Wu C, Weng G, Zhao Z, He X, Fu C, Sui Z, Huang SX: Bufalin Inhibits Cellular Proliferation and Cancer Stem Cell-Like Phenotypes via Upregulation of MiR-203 in Glioma. Cell Physiol Biochem 2017;44:671-681.

19 Shao Y, Gu W, Ning Z, Song X, Pei H, Jiang J: Evaluating the Prognostic Value of microRNA-203 in Solid Tumors Based on a Meta-Analysis and the Cancer Genome Atlas (TCGA) Datasets. Cell Physiol Biochem 2017;41:1468-1480.

20 Han R, Sun Q Wu J, Zheng P, Zhao G: Sodium Butyrate Upregulates miR-203 Expression to Exert AntiProliferation Effect on Colorectal Cancer Cells. Cell Physiol Biochem 2016;39:1919-1929.

21 Yi R, Poy MN, Stoffel M, Fuchs E: A skin microRNA promotes differentiation by repressing 'stemness'. Nature 2008;452:225-229. 


\section{Cellular Physiology Cell Physiol Biochem 2018;49:2333-2347 \begin{tabular}{l|l|l} 
DOI: 10.1159/000493834 & $\begin{array}{l}\text { O } 2018 \text { The Author(s). Published by S. Karger AG, Basel } \\
\text { www.karger.com/cpb }\end{array}$ \\
\hline and Biochemistry & Published online: 28 September 2018 .
\end{tabular} \\ Zhou et al.: MicroRNA-203 and Epidermal Stem Cells}

22 Sonkoly E, Wei T, Janson PC, Sääf A, Lundeberg L, Tengvall-Linder M, Norstedt G, Alenius H, Homey B, Scheynius A: MicroRNAs: novel regulators involved in the pathogenesis of psoriasis? PloS One 2007;2:e610.

23 Knight R, Melino G, Candi E: miR-203 represses stemness by repressing DeltaNp63. Cell Death Differ 2008;15:1187-1195.

24 Liu J, Xu Y, Shu B, Wang P, Tang J, Chen L, Qi S, Liu X, Xie J: Quantification of the differential expression levels of microRNA-203 in different degrees of diabetic foot. Int J Clin Exp Pathol 2015;8:13416-13420.

25 Pastar I, Khan AA, Stojadinovic O, Lebrun EA, Medina MC, Brem H, Kirsner RS, Jimenez JJ, Leslie C, TomicCanic M: Induction of specific microRNAs inhibits cutaneous wound healing. J Biol Chem 2012;287:2932429335.

-26 Zhang L, Stokes N, Polak L, Fuchs E: Specific microRNAs are preferentially expressed by skin stem cells to balance self-renewal and early lineage commitment. Cell Stem Cell 2011;8:294-308.

27 Yi R, Fuchs E: A miR image of stem cells and their lineages. Curr Top Dev Biol 2012;99:175.

28 Diao Y, Guo X, Jiang L, Wang G, Zhang C, Wan J, Jin Y, Wu Z: miR-203, a tumor suppressor frequently downregulated by promoter hypermethylation in rhabdomyosarcoma. J Biol Chem 2014;289:529-539.

29 Reiisi S, Esmaeili F, Shirazi A: Isolation, culture and identification of epidermal stem cells from newborn mouse skin. In vitro Cell Dev-An 2010;46:54-59.

30 Eckert RL, Adhikary G, Balasubramanian S, Rorke EA, Vemuri MC, Boucher SE, Bickenbach JR, Kerr C: Biochemistry of epidermal stem cells. BBA-Gen Subjects 2013;1830:2427-2434.

-31 Robert-Moreno A, Guiu J, Ruiz-Herguido C, Lopez ME, Ingles-Esteve J, Riera L, Tipping A, Enver T, Dzierzak E, Gridley T, Espinosa L, Bigas A: Impaired embryonic haematopoiesis yet normal arterial development in the absence of the Notch ligand Jagged1. EMBO J 2008;27:1886-1895.

-32 Shi Y, Shu B, Yang R, Xu Y, Xing B, Liu J, Chen L, Qi S, Liu X, Wang P: Wnt and Notch signaling pathway involved in wound healing by targeting c-Myc and Hes1 separately. Stem Cell Res Ther 2015;6:120.

-33 Bose A, Teh M-T, Mackenzie IC, Waseem A: Keratin k15 as a biomarker of epidermal stem cells. Int J Mol Sci 2013;14:19385-19398.

-34 Guo A, Jahoda CA: An improved method of human keratinocyte culture from skin explants: cell expansion is linked to markers of activated progenitor cells. Exp Dermatol 2009;18:720-726.

-35 Yang J, Su D, Li S, Gao L: The expression of alpha-smooth muscle actin in primary cultural fibroblasts of rats. Zhongguo ying yong sheng li xue za zhi= Zhongguo yingyong shenglixue zazhi= Chinese journal of applied physiology 2009;25:339-343.

-36 Al-Qattan MM, Abd-Elwahed MM, Hawary K, Arafah MM, Shier MK: Myofibroblast expression in skin wounds is enhanced by collagen III suppression. Biomed Res Int 2015;2015.

37 Guiu J, Shimizu R, D’Altri T, Fraser ST, Hatakeyama J, Bresnick EH, Kageyama R, Dzierzak E, Yamamoto M, Espinosa L: Hes repressors are essential regulators of hematopoietic stem cell development downstream of Notch signaling. J Exp Med 2013;210:71-84.

-38 Zhang Z, Yan R, Zhang Q Li J, Kang X, Wang H, Huan L, Zhang L, Li F, Yang S: Hes1, a Notch signaling downstream target, regulates adult hippocampal neurogenesis following traumatic brain injury. Brain Res 2014;1583:65-78.

-39 Soifer HS, Rossi JJ, Saetrom P: MicroRNAs in disease and potential therapeutic applications. Mol Ther 2007;15:2070-2079.

40 Shilo S, Roy S, Khanna S, Sen CK: MicroRNA in cutaneous wound healing: a new paradigm. DNA Cell Biol 2007;26:227-237.

41 Banerjee J, Chan YC, Sen CK: MicroRNAs in skin and wound healing. Physiol Genomics 2011;43:543-556.

42 Schneider M: MicroRNAs as novel players in skin development, homeostasis and disease. Brit J Dermatol 2012;166:22-28.

43 Teta M, Choi YS, Okegbe T, Wong G, Tam OH, Chong MM, Seykora JT, Nagy A, Littman DR, Andl T: Inducible deletion of epidermal Dicer and Drosha reveals multiple functions for miRNAs in postnatal skin. Development 2012;139:1405-1416.

44 Calabrese JM, Seila AC, Yeo GW, Sharp PA: RNA sequence analysis defines Dicer's role in mouse embryonic stem cells. P Natl Acad Sci USA 2007;104:18097-18102.

-45 Candi E, Amelio I, Agostini M, Melino G: MicroRNAs and p63 in epithelial stemness. Cell Death Differ 2015;22:12-21. 


\section{Cellular Physiology Cell Physiol Biochem 2018;49:2333-2347 \begin{tabular}{ll|l} 
and Biochemistry & $\begin{array}{l}\text { DOI: 10.1159/000493834 } \\
\text { Published online: } 28 \text { September } 2018\end{array}$ & $\begin{array}{l}\text { O 2018 The Author(s). Published by S. Karger AG, Basel } \\
\text { www.karger.com/cpb }\end{array}$ \\
\cline { 2 - 3 }
\end{tabular} \\ Zhou et al.: MicroRNA-203 and Epidermal Stem Cells}

46 Primo MN, Bak RO, Schibler B, Mikkelsen JG: Regulation of pro-inflammatory cytokines TNF $\alpha$ and IL24 by microRNA-203 in primary keratinocytes. Cytokine 2012;60:741-748.

47 Sun Y, Li X-Q, Sahbaie P, Shi X-Y, Li W-W, Liang D-Y, Clark JD: miR-203 regulates nociceptive sensitization after incision by controlling phospholipase A2 activating protein expression. Anesthesiology 2012;117:626-638.

48 Moffatt CE, Lamont RJ: Porphyromonas gingivalis induction of microRNA-203 expression controls suppressor of cytokine signaling 3 in gingival epithelial cells. Infect Immun 2011;79:2632-2637.

-49 Yuan Y, Zeng Z-Y, Liu X-H, Gong D-J, Tao J, Cheng H-Z, Huang S-D: MicroRNA-203 inhibits cell proliferation by repressing $\triangle$ Np63 expression in human esophageal squamous cell carcinoma. BMC Cancer 2011;11:57.

50 Broughton 2nd G, Janis JE, Attinger CE: The basic science of wound healing. Plast Reconstr Surg 2006;117:12S-34S.

51 Viticchie G, Lena A, Cianfarani F, Odorisio T, Annicchiarico-Petruzzelli M, Melino G, Candi E: MicroRNA-203 contributes to skin re-epithelialization. Cell Death Dis 2012;3:e435.

-52 Yang X, Wang J, Guo S-L, Fan K-J, Li J, Wang Y-L, Teng Y, Yang X: miR-21 promotes keratinocyte migration and re-epithelialization during wound healing. Int J Biol Sci 2011;7:685.

$53 \mathrm{Xu}$ J, Wu W, Zhang L, Dorset-Martin W, Morris MW, Mitchell ME, Liechty KW: The Role of MicroRNA-146a in the Pathogenesis of the Diabetic Wound-Healing Impairment. Diabetes 2012;61:2906-2912.

54 Bertero T, Gastaldi C, Bourget-Ponzio I, Imbert V, Loubat A, Selva E, Mari B, Hofman P, Barbry P, Meneguzzi G: miR-483-3p controls proliferation in wounded epithelial cells. FASEB J 2011;25:3092-3105. 